\title{
AÇÕES EM ENFERMAGEM NA NEFROLOGIA: 24 ANOS DE EXTENSÃO
}

\author{
ACTIONS NURSING NEPHROLOGY: 24 YEARS OF EXTENSION
}

\author{
Alana Duarte Flores ${ }^{1} \quad$ Vanessa Souza Lemes de Barros ${ }^{2} \quad$ Ricardo Aires da Silveira ${ }^{3}$ \\ Janina Neves Cardozo ${ }^{4} \quad$ Juliana Graciela Vestena Zillmer ${ }^{5} \quad$ Eda Schwartz $^{6}$
}

\begin{abstract}
RESUMO
O presente trabalho tem como objetivo descrever as ações de extensão na área de nefrologia desenvolvidas pela Enfermagem junto à comunidade e refletir sobre sua influência nos cenários de atuação e formação de enfermeiros com vista ao campo da nefrologia. Trata-se de um relato de experiência a partir da participação de acadêmicos de enfermagem no Projeto de Extensão "Internato em Enfermagem Nefrológica" no decorrer de seus 24 anos de vigência. A partir da análise de relatórios anuais identificaram-se dois eixos temáticos que permitiram descrever as ações desenvolvidas, o primeiro "A atuação do acadêmico de enfermagem na assistência ao paciente em diálise e seus familiares"; segundo, "Ações de prevenção da doença e promoção da saúde renal na comunidade".
\end{abstract}

Palavras-chave: Nefrologia. Enfermagem. Saúde.

\begin{abstract}
${ }^{1}$ Alana Duarte Flores - UFPEL - Brasil - Acadêmica de enfermagem pela Universidade Federal de Pelotas. Bolsista do projeto de pesquisa intitulado "Ações intersetoriais realizadas na visão da equipe de saúde e dos idosos na promoção da saúde para o envelhecimento do idoso rural". Voluntária no projeto de pesquisa "O processo de doação, captação e transplante de órgãos na perspectiva dos trabalhadores em saúde: um estudo qualitativo no Rio Grande do Sul”. Atuou como bolsista do projeto de extensão“ Internato em Enfermagem Nefrológica” alana_duarte2009@ hotmail.com; ${ }^{2}$ Vanessa Souza Lemes de Barros - UFPEL - Brasil - Acadêmica de Enfermagem pela Universidade Federal de Pelotas. Atuou com bolsista voluntária do projeto de extensão "Internato em Enfermagem Nefrológica. vanessahlems@gmail.com; ${ }^{3}$ Ricardo Aires da Silveira UFPEL - Brasil- Acadêmico de Enfermagem pela Universidade Federal de Pelotas. Atuou como bolsista do projeto de extensão "Internato em Enfermagem Nefrológica". ricardo.a.silveira@outlook.com; ${ }^{4}$ Janina Neves Cardozo - UFPEL - Brasil - Acadêmica de Enfermagem pela Universidade Federal de Pelotas. Atuou como bolsista voluntária do projeto de extensão "Internato em Enfermagem Nefrológica”. janina_neves@ hotmail.com; ${ }^{5}$ Juliana Graciela Vestena Zillmer - UFPEL - Brasil - Graduada em Enfermagem pela Universidade Federal de Pelotas e Mestre em Enfermagem pela UFPEL. Doutora em Enfermagem pela Universidade Federal de Santa Catarina. Professora Adjunta da Faculdade de Enfermagem da UFPEL. juzillmer@gmail.com; ${ }^{6}$ Eda Schwartz - UFPEL - Brasil - Possui graduação em Enfermagem pela Universidade Federal de Pelotas, mestrado em Enfermagem pela Universidade Federal de Santa Catarina e doutorado em Enfermagem pela Universidade Federal de Santa Catarina. Realizou Pos Doutorado na EEUSP em 2012. Professora Associada da UFPEL, docente do Programa de Pós-graduação em Enfermagem. eschwartz@terra.com.br
\end{abstract}




\section{ABSTRACT}

The present study aims at describing the extension actions in the area of nephrology developed in the community and reflecting on its influence in the scenarios of performing and training of nurses with a view to the field of nephrology. This is an experience report from the participation of nursing students in the Extension Project "Internship in Nephrological Nursing" during its 24 years of validity. From the analysis of annual reports, two thematic axes were identified, which allowed to describe the actions developed, the first one "The nursing student's role in the care of patients on dialysis and their relatives"; Second, "Actions of disease prevention and promoting renal health in the community".

Keywords: Nephrology. Health. Nursing.

\section{INTRODUÇÃO}

A doença renal crônica (DRC) se define como a perda da função renal de forma progressiva e irreversível, em que leva a pessoa doente a necessitar de uma terapia de substituição renal (BASTOS; KIRSZTAJN, 2011). O Sistema Único de Saúde (SUS) disponibiliza para as pessoas com DRC terapias que substituem o rim e realiza sua função, de acordo com a necessidade de cada indivíduo, sendo elas: hemodiálise, diálise peritoneal e transplante renal (BRASIL, 2014).

A hemodiálise tem como finalidade substituir a filtração renal, retirando resíduos que são prejudicais a saúde do sangue por meio de uma máquina (FRAZÃO et al, 2014). A diálise peritoneal é uma técnica fisiológica, no qual se utiliza o peritônio como filtro (BRUNNER; SUDDARTH, 2014). Já o transplante renal é a substituição do rim doente por um outro mediante doação do órgão de uma pessoa saudável, podendo ser de uma pessoa falecida ou viva com o objetivo de melhorar a qualidade de vida (BRASIL, 2014). Mediante o apresentado, os tratamentos disponíveis para a doença, como diálise e transplante renal, ocasionam mudanças e limitações físicas, psicológicas, sexuais, sociais e familiares no viver diário (OLIVEIRA et al., 2016; SANTOS et al., 2016; ZILLMER; DA SILVA; MERCADO-MARTINEZ, 2015).

As pessoas com DRC expressam sentimentos de incapacidade, negatividade e culpa devido ao receio do prognóstico, da dependência financeira e alterações na autoimagem, autoestima e autocuidado (SILVA et al., 2011). Além destes sentimentos e alterações dificuldades da descoberta da doença e no dia a dia para a realização do tratamento, seja pela busca do serviço, pela insuficiência de vagas, pela falta de equipamentos de diálise, ou devido ao deslocamento de quem reside na área rural, assim como a questão financeira para arcar com custos do transporte, alimentação medicamentos e adaptações na residência para 
continuidade do tratamento (MARTINEZ et al., 2015). No entanto, entendem que o tratamento é necessário para sua sobrevivência (SILVA et al., 2011).

Frente ao adoecimento da pessoa com DRC, enquanto importante problema de saúde pública, faz-se necessário possibilitar a acadêmicos de enfermagem, aproximação com o campo da Nefrologia, com o objetivo de sensibilizá-los e instrumentalizá-los a compreender experiências e obstáculos destas pessoas e seus familiares com a finalidade de subsidiar práticas da atenção à saúde dispensada pelo SUS.

Entre as atividades desenvolvidas pelo enfermeiro está a educação em saúde, a qual pode ser realizada desde a atenção primária até o nível terciário de saúde, em parceria entre profissional, paciente e família. Desta forma, o enfermeiro possui importante papel como cuidador e educador, pois, além do compromisso ético e profissional que o torna responsável por sistematizar e incentivar o autocuidado, desenvolver atividades educativas de promoção de saúde, reduzir a incidência da DRC, assim como buscar o bem estar e melhoria da qualidade de vida do paciente (SANTOS et al., 2017).

Diante do apresentado, o presente artigo tem como objetivo descrever as ações de extensão na área de nefrologia desenvolvidas junto à comunidade mediante o Projeto de Extensão "Internato em Enfermagem Nefrológica” no decorrer de seus 24 anos de vigência. Além disto, refletir sobre sua influência nos cenários de atuação e formação de enfermeiros com vista à área da nefrologia no contexto do Sistema Único de Saúde.

\section{METODOLOGIA}

Trata-se de um relato de experiência a partir da participação de acadêmicos de enfermagem no Projeto de Extensão "Internato em Enfermagem Nefrológica" no decorrer de seus 24 anos de vigência. A modalidade de publicações técnico-científicas inclui os relatos de experiência como documento utilizado para relatar formalmente resultados ou progressos obtidos em investigação de pesquisa e desenvolvimento, bem como forma de descrever a situação de uma questão técnica ou científica (ASSOCIAÇÃO BRASILEIRA DE NORMAS TÉCNICAS, 2011).

O Projeto de Extensão "Internato em Enfermagem Nefrológica” é desenvolvido pela Faculdade de Enfermagem (FEn) da Universidade Federal de Pelotas (UFPel), tendo seu início no ano de 1992 e, desde então vem atuando nos serviços de nefrologia, na comunidade, escolas e unidades básicas de saúde vinculadas à Universidade. Tal projeto originou-se a partir da articulação de docentes, acadê- 
micos da FEn da UFPel e profissionais dos serviços de nefrologia de Pelotas, Rio Grande do Sul/Brasil.

As atividades em questão foram realizadas por acadêmicos de enfermagem do primeiro ao décimo semestre, bolsistas e voluntários, supervisionados por facilitadores enfermeiros e docentes nos distintos cenários de atuação do referido projeto. A articulação de atividades é realizada de acordo com o semestre dos discentes, do primeiro ao quarto semestre, nos quais desenvolveram atividades na comunidade, escolas e unidades básicas de saúde. Já os discentes a partir do quinto, desenvolveram além de atividades na comunidade, atividades nos hospitais e serviços de nefrologia.

As atividades desenvolvidas são planejadas e executadas considerando o cenário e o público alvo, para os quais os discentes são orientados e capacitados pelo professor coordenador do projeto previamente mediante encontros quinzenais. O projeto possui carga horária total de 160 horas anuais, portanto os discentes necessitam cumprir a partir de cinco horas semanais e totalizar no mínimo 120 horas para receber o certificado de participação. Anualmente há um edital de seleção para concorrer à bolsa de extensão, cujo discente selecionado possui carga horária mínima semanal de 20 horas e também conta com a participação de acadêmicos voluntários.

Para a construção do presente trabalho foram compilados dados oriundos de relatórios anuais do projeto, por meio dos quais, foi possível organizar e descrever as atividades realizadas durante o período de 1992 a 2016. O processo de análise consistiu na leitura repetida dos textos e imersão nos dados dos relatórios para ter uma visão do conjunto e permitir a compreensão.

\section{RESULTADOS}

O Projeto ao longo dos anos teve em sua coordenação majoritariamente o mesmo docente como responsável, com participação em média de 300 alunos. As articulações com outros cursos ocorreram de forma pontual.

A partir da análise de relatórios anuais identificaram-se dois eixos temáticos que permitiram descrever as ações desenvolvidas mediante o Projeto de Extensão: "Atuação dos acadêmicos de enfermagem na assistência ao paciente em diálise e seus familiares"; e "Ações de prevenção da doença e promoção da saúde renal na comunidade". 


\section{ATUAÇÃO DOS ACADÊMICOS DE ENFERMAGEM NA ASSISTÊNCIA À PES- SOA EM DIÁLISE E SEUS FAMILIARES}

As atividades mediante o projeto de extensão permitiram conhecer o contexto do serviço de nefrologia, enquanto área física, sua dinâmica e funcionamento; o processo de trabalho da equipe de saúde; os tratamentos disponíveis pelo SUS e suas especificidades, sendo eles tratamento conservador, a diálise peritoneal e a hemodiálise. Além disto, permitiu aproximar os acadêmicos dos familiares da pessoa com DRC buscando desenvolver uma assistência voltada a necessidade da família. Esta aproximação ocorreu mediante a inserção dos acadêmicos no serviço de nefrologia a partir do acompanhamento dos familiares na sala de espera, assim como dos pacientes durante as sessões de diálise.

O serviço de nefrologia desenvolve cuidados a pacientes e familiares que são cadastrados e acompanhados pelo serviço o qual era referência no município em diálise peritoneal. A estrutura física do local contém sala recepção, local de pesagem dos pacientes, sala de procedimentos, consultório médico e de enfermagem, a sala de hemodiálise, sala de diálise peritoneal, área de utilidades, tratamento de água por osmose reversa para a purificação de água em diálise, atendimento multidisciplinar. Além disso, proporciona tratamento para pacientes graves, na Unidade de Terapia Intensiva (UTI), o seu funcionamento é 24 horas, durante os sete dias da semana.

Os acadêmicos de enfermagem tiveram a possibilidade de conhecer e compreender os cuidados necessários a homens e mulheres, com distintas idades e condição socioeconômica que experienciam a DRC. Evidenciaram implicações na vida diária, alterando sua qualidade, incluindo restrição na dieta e hídrica, lazer, trabalho, relações sociais; além disso, são submetidos a procedimentos invasivos como punções venosas frequentes, exames laboratoriais e consultas periódicas.

A sistematização da assistência de enfermagem foi desenvolvida pelos acadêmicos com ênfase no histórico de enfermagem, sendo construído um específico para o serviço de diálise para aplicação, evolução e prescrição de enfermagem. Os acadêmicos acompanharam o enfermeiro do serviço na capacitação de pacientes e familiares para a realização da terapia no domicílio.

A assistência do acadêmico de enfermagem a pacientes em hemodiálise aconteceu também mediante a realização de procedimentos, tais como: verificação de sinais vitais, pesagem antes e a após a diálise, realização de exame físico, desenvolvimento da sistematização da assistência de enfermagem, realização de curativos em fístula arteriovenosa e cateter, auxiliar durante o preparo das máquinas de hemodiálise, acompanhar a desinfecção dos capilares pós hemodi- 
álise, organização dos materiais nas salas de diálise, e transporte em cadeira de rodas.

Quanto à atuação do acadêmico de enfermagem na assistência ao paciente em diálise peritoneal ocorreu mediante a realização de consultas de enfermagem à paciente em diálise peritoneal contínua, como verificação de sinais vitais, pesagem antes da consulta, ações educativas para o autocuidado do cateter de diálise peritoneal, assim como cuidados com a alimentação, higiene, curativos e materiais necessários para a realização de tal tratamento no domicílio.

A assistência de enfermagem ao paciente com DRC e sua família, ocorreu de maneira integral, realizando orientações e suprindo dúvidas sobre os tratamentos e doença. Entre as orientações estavam os cuidados com a alimentação, ingesta hídrica, e higiene pessoal, além de cuidados com o cateter de diálise e ou a fistula arteriovenosa, tipos de diálise, e o transplante renal. Além disto, foram desenvolvidos cuidados também para outras doenças crônicas como hipertensão, diabetes mellitus, hepatite B ou C e HIV (Vírus da Imunodeficiência Humana).

Com a finalidade de promover a autoestima das pessoas com DRC, foram organizadas festas em datas comemorativas, dia da beleza, homenagem da clínica a pacientes aniversariantes, e algumas sessões de musicoterapia no serviço de nefrologia. Para os familiares foi possível realizar a escuta terapêutica, atividades de educação em saúde na sala de espera, com orientações sobre a doença e tratamentos ambulatorial e domiciliar.

\section{AÇÕES DE PREVENÇÃO DA DOENÇA E PROMOÇÃO DA SAÚdE RENAL NA COMUNIDADE}

Os acadêmicos ao longo da atuação dos 24 anos junto ao projeto de extensão, desenvolveram atividades junto à comunidade mediante a educação em saúde, com o objetivo de sensibilizar a população para promoção da saúde renal e prevenção da DRC; além de ações de doação de órgãos e tecidos para transplante, principalmente o renal. Os cenários de atuação foram escolas, unidades básicas de saúde e praças públicas de Pelotas, incluindo o Campus Porto da UFPel.

No contexto escolar foram desenvolvidas atividades com crianças e adolescentes com o intuito de sensibilizá-los a adotarem um estilo de vida saudável desde a infância. Tratam-se de escolas públicas, localizadas na periferia de Pelotas, onde as ações foram desenvolvidas com crianças a partir dos seis anos de idade do primeiro ao sexto ano do ensino fundamental. Os acadêmicos buscaram informá-los sobre a prevenção da doença e a promoção da saúde renal, tendo como base as oito regras de ouro disponibilizadas pela Sociedade Brasileira de 
Nefrologia, sendo elas: realizar exercício físico, como caminhadas, andar de bicicleta, entre outras; controlar glicemia e pressão arterial, ingerir líquidos, evitar tabaco e bebidas alcoólicas, evitar automedicação, consultar o médico regularmente e por fim, manter uma alimentação saudável, onde explicou-se aos alunos sobre a diferença de alimentos naturais e industrializados, além das três principais refeições durante o dia que são necessárias para se ter uma vida saudável (SOCIEDADE BRASILEIRA DE NEFROLOGIA, 2016).

Distintas metodologias e recursos materiais foram utilizados para desenvolver as atividades, considerando a idade das crianças e dos adolescentes. Para as crianças utilizou-se o teatro com fantoches, desenhos que ilustravam alimentos naturais e industrializados. Já para adolescentes a utilização de recursos áudio visuais auxiliaram na discussão do tema, despertando o interesse e questionamentos dos mesmos. Ao final de cada atividade, utilizaram-se instrumentos de avaliação, com o objetivo de identificar se haviam entendido o que foi discutido. Para os adolescentes foram realizadas perguntas que necessitariam ser respondidas no decorrer do encontro considerando o tema abordado; já para as crianças o uso de desenhos para serem coloridos.

Nas Unidades Básicas de Saúde (UBS) foram realizadas atividades de educação em saúde, sobre a DRC no grupo de pessoas com Hipertensão Arterial Sistêmica (HAS) e Diabetes mellitus (DM) com a utilização de recursos audiovisuais, e distribuição de folders explicativos sobre como prevenir a doença. O contato com a UBS ocorreu por meio de uma conversa prévia com o enfermeiro coordenador do serviço de saúde, o qual informou os dias e os horários em que ocorriam as reuniões do grupo de acompanhamento de hipertensos e diabéticos. Este grupo é chamado de HIPERDIA, composto por mulheres de idades distintas, que variam entre 60 e 70 anos.

A maioria das participantes do HIPERDIA questionou sobre os sinais e sintomas da DRC, assim como dos cuidados necessários para preveni-la a partir de suas experiências com outras doenças, como, por exemplo, o diabetes mellitus e a hipertensão arterial. Alguns questionamentos estavam relacionados sobre qual era a causa da retenção de líquidos, pois algumas participantes relataram que se sentiam "inchaço" nas pernas, pés e mãos. No decorrer da apresentação dos temas referentes a cada patologia, havia questionamentos sobre o porquê ocorriam determinados sinais e sintomas, bem como, detalhavam o que sentiam em cada um deles e o que faziam para amenizá-los.

Quanto às ações nos espaços públicos, estas foram realizadas com o objetivo de sensibilizar a população sobre o tema doação de órgãos e tecidos para transplantes, sendo um deles o transplante renal. Os locais para tal atividade foram praças e Mercado Público Central de Pelotas e o Campus Porto da UFPel. Além destes, foram realizadas caminhadas no dia mundial da doação de órgãos, 
até a Praia do Laranjal, tendo como objetivo comover e mobilizar a população local sobre o tema.

As ações em espaços públicos contaram com a participação de acadêmicos de enfermagem, docentes, integrantes das Comissões Intra-Hospitalar de Doação de Órgãos e Tecidos para Transplante (CIHDOTT) enfermeiros, médicos e técnicos de enfermagem. Durante esta atividade, pode-se evidenciar que muitos jovens abordados possuíam pouca ou nenhuma informação sobre o processo de doação de órgãos, bem como, não sabiam que para serem doadores, a família deveria ser informada sobre tal decisão. Outro ponto destacado é que as pessoas com maior idade não se permitam serem abordadas e falar sobre o assunto. No entanto, a atividade possibilitou aos acadêmicos entender a opinião da população a respeito da doação de órgãos e, promover o diálogo com a comunidade sobre o tema.

Ao desenvolver a referida ação, esta se constitui em um momento propício para vincular o grupo de acadêmicos e docentes do Curso de Enfermagem, com as CIHDOTTs e a população em geral, fortalecendo a ação desenvolvida.

\section{DISCUSSÃO}

As pessoas ao depararem-se com o impacto da doença DRC e do tratamento, tornam-se indivíduos fragmentados, passando a viver com uma condição crônica de saúde (VIEIRA et al., 2011). No decorrer de sua experiência de adoecer, ocorrem mudanças na vida diária, tida como radical, com visitas aos serviços de saúde, uso de cateteres, limitações hídricas, modificações na dieta, alterações na autoimagem e autoestima, dentre outras (ZILLMER; DA SILVA; MERCADO-MARTINEZ, 2015; SILVA et al., 2013). Tais mudanças podem influenciar na adesão ao tratamento, na aceitação e cuidado de si, nas relações interpessoais com familiares e no convívio social (SILVA et al., 2013).

Mediante o projeto, a aproximação ocorreu também com a comunidade, crianças, adolescentes e pessoas que podem vir a desenvolver a DRC, sensibilizando-as para a adoção de hábitos saudáveis, com vistas a preveni-la, promovendo a saúde renal. Considerou-se o espaço da escola e o de grupos de pessoas com HAS e DM nas UBS, como espaços férteis para as ações, uma vez que, este público pode ter um melhor entendimento sobre a importância em adotar novos hábitos de vida ao dialogar e compartilhar com seus pares sobre tal tema e, por conseguinte reduzir os índices de doenças crônicas, com ênfase na DRC, no contexto brasileiro.

O acolhimento da pessoa com doença renal em início de hemodiálise ou diálise peritoneal fica sob a responsabilidade do enfermeiro pois é ele que, através da sua intervenção, tem um melhor conhecimento da pessoa e da sua 
história, estabelecendo com ela um elo de ligação com o serviço, além de uma relação de proximidade e criação de vínculo ( FERNANDES, 2018). Diante disso, evidencia-se a importância do papel do enfermeiro em ser um dos apoios da pessoa com doença renal crônica, em acolhê-la desde a descoberta do diagnóstico até a evolução do tratamento, ofertar informações a acerca da doença, bem como influenciar na sua segurança e autocuidado.

$\mathrm{O}$ acadêmico de enfermagem, vinculado ao projeto, teve a oportunidade de estar acompanhando o paciente durante seu tratamento, conhecer sua história e estabelecer uma relação de proximidade e confiança, o que contribui significativamente para o sucesso de todo processo. Além disso, pôde vivenciar como a teoria é aplicada na prática e os cuidados necessários para cada paciente.

Segundo Silva et al. (2015), o enfermeiro deve atuar na prevenção e na progressão da doença renal, visando ações para atender as necessidades das pessoas acometidas pela enfermidade, a fim de proporcionar melhor qualidade de vida, bem como, detectar os grupos de risco, com ações de prevenção por meio de intervenções que impeçam o desenvolvimento da doença renal.

Integrar projetos de pesquisa e extensão de Instituições de Ensino Superior (IES) proporciona aos acadêmicos de graduação novas descobertas e possibilidades que contribuem para a formação profissional (SANTOS; ANJOS; ALMEIDA, 2015). Ao longo destes anos mediante as ações desenvolvidas pelo projeto foi possível compartilhar conhecimento, experiências e práticas sobre temas que constituem a área de Nefrologia. Tais ações desenvolvidas foram divulgadas, com maior ênfase, em eventos de extensão promovidos pela UFPel, compartithando o caminhar do projeto com a comunidade acadêmica.

\section{CONSIDERAÇÕES FINAIS}

As ações de extensão realizadas a partir do projeto "Internato em Enfermagem Nefrológica", contribuíram para a formação de acadêmicos de enfermagem, educação em saúde da comunidade, com vistas a considerar o campo de Enfermagem em Nefrologia como parte da formação do enfermeiro para o SUS.

O projeto possibilitou aos acadêmicos uma aproximação com pessoas que vivenciam a doença renal e diálise, familiares e profissionais de saúde dos serviços. Desenvolver ações de educação em saúde e de assistência neste contexto promoveu refletir sobre o cuidado desenvolvido a este grupo, com vista a um cuidado menos biológico e mais integral ao considerar as dimensões cultural, social e espiritual, e a família como parte do cuidado. Entende-se que as atividades desenvolvidas nos serviços de diálise foram importantes para as pessoas com DRC, uma vez que, estas promoveram o conhecimento da pessoa sobre a doença, 
auxiliou no seu entendimento diante de complicação, assim como a promoção do autocuidado e consequentemente na melhora da qualidade de vida.

A proposta do projeto de extensão "Internato em Enfermagem Nefrológica" foi proporcionar aos acadêmicos de enfermagem uma aproximação à área de nefrologia, com maior ênfase no trabalho da enfermagem frente a este campo de conhecimento. Ao analisar as ações desenvolvidas mediante a experiência dos mesmos, pode-se afirmar que os objetivos do referido projeto de extensão foram desenvolvidos e alcançados.

Este projeto concedeu à comunidade benefícios, uma vez que proporcionou compartilhar informação quanto a prevenção e promoção da saúde renal por meio de atividades educativas pautadas em referenciais científicos atualizados sobre a temática; a ações voltadas para a assistência da pessoa com DRC em diálise e família. De maneira geral, o projeto devolveu à comunidade aquilo que é estudado e discutido na academia, atendendo as demandas de saúde sobre o tema. Já para o âmbito acadêmico, possibilitou o estudo e a aproximação da temática. Assim como o aprofundamento sobre a doença e implementação da Sistematização da Assistência de Enfermagem (SAE) específica ao serviço.

As percepções sobre as pessoas e familiares que convivem com tal enfermidade, puderam ser ampliadas e as ações direcionadas a estes grupos foram formuladas e realizadas utilizando conhecimentos adquiridos através de publicações sobre o tema, alcançando a finalidade de qualificar cada vez mais os acadêmicos, os tornando futuramente enfermeiros ativos na especialidade nefrologia.

\section{REFÊRENCIAS}

ABNT. NBR 10719: Informação e documentação - Relatório técnico e/ou científico - Apresentação. Rio de Janeiro, 2011.

BASTOS, M. G.; KIRSZTAJN, G.M. Doença renal crônica: importância do diagnóstico precoce, encaminhamento imediato e abordagem interdisciplinar estruturada para melhora do desfecho em pacientes ainda não submetidos à diálise. Jornal Brasileiro de Nefrologia, v. 33, n. 1, p. 93-108, 2011. Disponível em: <http:// www.scielo.br/scielo.php?pid=S010128002011000100013\&script=sci_abstract\&tlng=pt>. Acesso em: 21 set. 2016.

BRASIL. Ministério da Saúde. Secretária de Atenção a Saúde. Departamento de Atenção Especializada e Temática. Diretrizes clínicas para o cuidado ao paciente com doença renal crônica - DRC no Sistema Único de Saúde. Brasília: 
Ministério da Saúde, 2014. Disponível em: <http://sonerj.org.br/wp-content/uploads/2014/03/diretriz-cl-nica-drc-versao-final2.pdf>. Acesso em: 21 set. 2016.

CHEEVER, Kerry H.; HINKLE, Janice L. BRUNNER \& SUDDARTH: tratado de enfermagem médico-cirúrgica. 12. ed. Rio de Janeiro: Guanabara Koogan, 2014.

FERNANDES, M. I. D. Intervenção para a promoção da adesão ao regime terapêutico da pessoa em início de hemodiálise: uma pesquisa qualitativa. Revista Pesquisa Qualitativa, v. 6, n. 10, p. 60-75, 2018. Disponível em: <http://rpq.revista. sepq.org.br/index.php/rpq/article/view/206/107>. Acesso em: 8 maio.2018.

FRAZÃO, C. M. F. Q. et al. Cuidados de enfermagem ao paciente renal crônico em hemodiálise. Revista da Rede de Enfermagem do Nordeste, v. 14, n. 4, p. 701-709, 2014. Disponível em: <http://www.repositorio.ufc.br/bitstream/riufc/10441/1/2014_art_albclira.pdf>. Acesso em: 15 nov. 2016.

LINS, L. et al. Extensão universitária e inclusão social de estudantes do ensino médio público. Revista Trabalho, Educação e Saúde, v. 12, n. 3, p. 779-694, 2014. Disponível em: <http://www.scielo.br/scielo.php?script=sci_arttext\&pi$d=$ S1981-77462014000300679 > . Acesso em: 17 set. 2016.

MARTINEZ, F. J. M. et al. Vivendo com insuficiência renal: obstáculos na terapia da hemodiálise na perspectiva das pessoas doentes e suas famílias, Physis Revista de Saúde Coletiva, v. 25, n. 1, p. 59-74, 2015. Disponível em: <http://www. scielo.br/pdf/physis/v25n1/0103-7331-physis-25-01-00059.pdf>. Acesso em: 8 mar. 2017.

OLIVEIRA, V. A. et al. Relações familiares de mulheres em hemodiálise. Revista de Atenção à Saúde, São Caetano do Sul, v. 14, n. 47, p. 36-42, 2016. Disponível em: <http://seer.uscs.edu.br/index.php/revista_ciencias_saude/article/viewFile/3283/pdf $>$. Acesso em: 15 dez. 2016.

DOS SANTOS, B. P. et al. Foi/não foi tudo o que pensava: facilidades e dificuldades após o transplante renal. Revista Gaúcha de Enfermagem, v. 37, n. 3, p. 60135, 2016. Disponível em: <http://www.scielo.br/pdf/rgenf/v37n3/0102-6933-rgenf-1983-144720160360135.pdf>. Acesso em: 10 nov. 2016.

SANTOS, V. C.; ANJOS, K. F.; ALMEIDA, O. S. A percepção de formandos sobre a pesquisa em enfermagem no curso de graduação. Revista de Enfermagem da UFSM, v. 3, n. 1, p. 144-154, 2013. Disponível em: <https://periodicos.ufsm.br/reufsm/article/view/7746>. Acesso em: 10 maio 2017.

SANTOS, J. K. F. M. et al. Estratégias da atenção básica na doença renal crônica: 
a importância do diagnóstico precoce. Rev. Saúde .Com, v. 2, n. 13, p. 863-870, 2017.

SILVA, A. et al. A ação do enfermeiro na prevenção de doença renais crônicas: uma revisão integrativa. SANARE, v. 14, n. 2, p. 148-155, 2015. Disponível em: <https://sanare.emnuvens.com.br/sanare/article/viewFile/840/511 >. Acesso em: 06 maio 2018.

SILVA, M. et al. A sala de espera como espaço de educação e promoção de saúde à pessoa com insuficiência renal crônica em hemodiálise. Revista Online de Pesquisa Cuidado é Fundamental, v. 5, n. 3, p. 253-263, 2013. Disponível em: <http://pesquisa.bvsalud.org/brasil/resource/pt/bde-25117>. Acesso em: 12 nov. 2016.

SOCIEDADE BRASILEIRA DE NEFROLOGIA. Dia Mundial do Rim - 2016. 2015. Disponível em: <https://sbn.org.br/events/dia-mundial-do-rim-2016>. Acesso em: 15 nov. 2016.

VIEIRA, T. G. et al. Práticas de educação em saúde para pacientes que realizam diálise peritoneal no domicílio. Revista Contexto e Saúde, v. 10, n. 20, p. 12171222, 2011. Disponível em: <https://webcache.googleusercontent.com/search?q=cache:QW6ehldXsTAJ:https://www.revistas.unijui.edu.br/index.php/contextoesaude/article/download $/ 1777 / 1478+\& \mathrm{~cd}=1 \& \mathrm{qh}=\mathrm{pt}-\mathrm{BR} \& \mathrm{ct}=\mathrm{clnk \& gl=br}>$. Acesso em: 15 set. 2016.

ZILLMER, J. G. V.; DA SILVA, D. V. G.; MERCADO-MARTINEZ, F. J. Sobre o meu corpo mando eu! O manejo do tratamento na vida das pessoas em diálise peritoneal. CIAIQ2015, v. 1, p. 303-309, 2015. Disponível em: <http://proceedings.ciaiq. org/index.php/ciaiq2015/article/view/72/69>. Acesso em: 12 nov. 2016.

Data de recebimento: 22 de fevereiro de 2018.

Data de aceite para publicação: 09 de maio de 2018. 